Pacific Journal of Mathematics

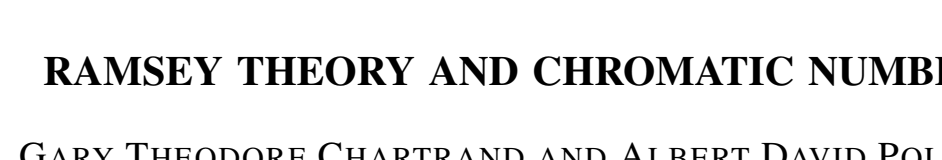




\title{
RAMSEY THEORY AND CHROMATIC NUMBERS
}

\author{
Gary Chartrand and Albert D. Polimeni
}

Let $\chi(G)$ denote the chromatic number of a graph $G$. For positive integers $n_{1}, n_{2}, \cdots, n_{k}(k \geqq 1)$ the chromatic Ramsey number $\chi\left(n_{1}, n_{2}, \cdots, n_{k}\right)$ is defined as the least positive integer $p$ such that for any factorization $K_{p}=\bigcup_{i=1}^{k} G_{i}, \chi\left(G_{i}\right) \geqq n_{i}$ for at least one $i, 1 \leqq i \leqq k$. It is shown that $\chi\left(n_{1}, n_{2}, \cdots, n_{k}\right)=$ $1+\prod_{i=1}^{k}\left(n_{i}-1\right)$. The vertex-arboricity $a(G)$ of a graph $G$ is the fewest number of subsets into which the vertex set of $G$ can be partitioned so that each subset induces an acyclic graph. For positive integers $n_{1}, n_{2}, \cdots, n_{k}(k \geqq 1)$ the vertexarboricity Ramsey number $a\left(n_{1}, n_{2}, \cdots, n_{k}\right)$ is defined as the least positive integer $p$ such that for any factorization $K_{p}=$ $\mathrm{U}_{i=1}^{k} G_{i}, a\left(G_{i}\right) \geqq n_{i}$ for at least one $i, 1 \leqq i \leqq k$. It is shown that $a\left(n_{1}, n_{2}, \cdots, n_{k}\right)=1+2 k \prod_{i=1}^{k}\left(n_{i}-1\right)$.

Introduction. The classical Ramsey number $r(m, n)$, for positive integers $m$ and $n$, is the least positive integer $p$ such that for any graph $G$ of order $p$, either $G$ contains the complete graph $K_{m}$ of order $m$ as a subgraph or the complement $\bar{G}$ of $G$ contains $K_{n}$ as a subgraph. More generally, for $k(\geqq 1)$ positive integers $n_{1}, n_{2}, \cdots, n_{k}$, the Ramsey number $r\left(n_{1}, n_{2}, \cdots, n_{k}\right)$ is defined as the least positive integer $p$ such that for any factorization $K_{p}=G_{1} \cup G_{2} \cup \cdots \cup G_{k}$ (i.e., the $G_{i}$ are spanning, pairwise edge-disjoint, possibly empty subgraphs of $K_{p}$ such that the union of the edge sets of the $G_{i}$ equals the edge set of $\left.K_{p}\right), G_{i}$ contains $K_{n_{i}}$ as a subgraph for at least one $i, 1 \leqq i \leqq$ $k$. It is known (see [5]) that all such Ramsey numbers exist; however, the actual values of $r\left(n_{1}, n_{2}, \cdots, n_{k}\right), k \geqq 1$, are known in only seven cases (see $[2,3]$ ) for which $\min \left\{n_{1}, n_{2}, \cdots, n_{k}\right\} \geqq 3$.

A clique in a graph $G$ is a maximal complete subgraph of $G$. The clique number $\omega(G)$ is the maximum order among the cliques of $G$. The Ramsey number $r\left(n_{1}, n_{2}, \cdots, n_{k}\right)$ may be alternatively defined as the least positive integer $p$ such that for any factorization $K_{p}=$ $G_{1} \cup G_{2} \cup \cdots \cup G_{k}, \omega\left(G_{i}\right) \geqq n_{i}$ for at least one $i, 1 \leqq i \leqq k$.

The foregoing observation suggests the following definition. Let $f$ be a graphical parameter, and let $n_{1}, n_{2}, \cdots, n_{k}, k \geqq 1$ be positive integers. The $f$-Ramsey number $f\left(n_{1}, n_{2}, \cdots, n_{k}\right)$ is the least positive integer $p$ such that for any factorization $K_{p}=G_{1} \cup G_{2} \cup \cdots \cup G_{k}$, $f\left(G_{i}\right) \geqq n_{i}$ for at least one $i, 1 \leqq i \leqq k$. Hence, $\omega\left(n_{1}, n_{2}, \cdots, n_{k}\right)=$ $r\left(n_{1}, n_{2}, \cdots, n_{k}\right)$, i.e., the $\omega$-Ramsey number is the Ramsey number.

The object of this paper is to investigate $f$-Ramsey numbers for two graphical parameters $f$, namely chromatic number and vertexarboricity. 
Chromatic Ramsey numbers. The chromatic number $\chi(G)$ of a graph $G$ is the fewest number of colors which may be assigned to the vertices of $G$ so that adjacent vertices are assigned different colors. For positive integers $n_{1}, n_{2}, \cdots, n_{k}$, the chromatic Ramsey number $\chi\left(n_{1}, n_{2}, \cdots, n_{k}\right)$ is the least positive integer $p$ such that for any factorization $K_{p}=G_{1} \cup G_{2} \cup \cdots G_{k}, \chi\left(G_{i}\right) \geqq n_{i}$ for some $i, 1 \leqq i \leqq$ $k$. The existence of the numbers $\chi\left(n_{1}, n_{2}, \cdots, n_{k}\right)$ is guaranteed by the fact that $\chi\left(n_{1}, n_{2}, \cdots, n_{k}\right) \leqq r\left(n_{1}, n_{2}, \cdots, n_{k}\right)$. We are now prepared to present a formula for $\chi\left(n_{1}, n_{2}, \cdots, n_{k}\right)$. We begin with a lemma.

Lemma. If $G=G_{1} \cup G_{2} \cup \cdots \cup G_{k}$, then

$$
\chi(G) \leqq \sum_{i=1}^{k} \chi\left(G_{i}\right) \text {. }
$$

Proof. For $i=1,2, \cdots, k$, let a $\chi\left(G_{i}\right)$-coloring be given for $G_{i}$. We assign to a vertex $v$ of $G$ the color $\left(c_{1}, c_{2}, \cdots, c_{k}\right)$, where $c_{i}$ is the color assigned to $v$ in $G_{i}$. This produces a coloring of $G$ using at most $\prod_{i=1}^{k} \chi\left(G_{i}\right)$ colors; hence, $\chi(G) \leqq \prod_{i=1}^{k} \chi\left(G_{i}\right)$.

THEOREM 1. For positive integers $n_{1}, n_{2}, \cdots, n_{k}$,

$$
\chi\left(n_{1}, n_{2}, \cdots, n_{k}\right)=1+\prod_{i=1}^{k}\left(n_{i}-1\right) .
$$

Proof. The result is immediate if $n_{i}=1$ for some $i$; hence, we assume that $n_{i} \geqq 2$ for all $i, 1 \leqq i \leqq k$. First, we verify that

$$
\chi\left(n_{1}, n_{2}, \cdots, n_{k}\right) \leqq 1+\prod_{i=1}^{k}\left(n_{i}-1\right) .
$$

Let $p=1+\Pi_{i=1}^{k}\left(n_{i}-1\right)$, and assume there exists a factorization $K_{p}=G_{1} \cup G_{2} \cup \cdots \cup G_{k}$ such that $\chi\left(G_{i}\right) \leqq n_{i}-1$ for each $i=1,2, \cdots, k$. Then by the Lemma, it follows that

$$
1+\prod_{i=1}^{k}\left(n_{i}-1\right)=\chi\left(K_{p}\right) \leqq \prod_{i=1}^{k} \chi\left(G_{i}\right) \leqq \prod_{i=1}^{k}\left(n_{i}-1\right),
$$

which produces a contradiction. Thus, in any factorization $K_{p}=$ $G_{1} \cup G_{2} \cup \cdots \cup G_{k}$ for $p=1+\prod_{i=1}^{k}\left(n_{i}-1\right)$, we have $\chi\left(G_{i}\right) \geqq n_{i}$ for at least one $i, 1 \leqq i \leqq k$.

In order to show that

$$
\chi\left(n_{1}, n_{2}, \cdots, n_{k}\right) \geqq 1+\prod_{i=1}^{k}\left(n_{i}-1\right),
$$

we exhibit a factorization $K_{N_{k}}=G_{1} \cup G_{2} \cup \cdots \cup G_{k}$, where $N_{k}=$ 
$\Pi_{i=1}^{k}\left(n_{i}-1\right)$ and $\chi\left(G_{i}\right) \leqq n_{i}-1$ for $i=1,2, \cdots, k$. The factorization is accomplished by employing induction on $k$. For $k=1$, we simply observe that $\chi\left(K_{N_{1}}\right)=\chi\left(K_{n_{1}-1}\right)=n_{1}-1$. Assume there exists a factorization $K_{N_{k-1}}=H_{1} \cup H_{2} \cup \cdots \cup H_{k-1}$ such that $\chi\left(H_{i}\right) \leqq n_{i}-1$ for $i=1,2, \cdots, k-1$. Let $F$ denote $n_{k}-1$ (pairwise disjoint) copies of $K_{N_{k-1}}$ and define $G_{k}$ by $G_{k}=\bar{F}$. Thus, $\bar{G}_{k}$ contains $n_{k}-1$ pairwise disjoint copies of $H_{i}$ for $i=1,2, \cdots, k-1$, which we denote by $G_{i}$. Hence, $K_{N_{k}}=G_{1} \cup G_{2} \cup \cdots \cup G_{k}$, where $\chi\left(G_{i}\right) \leqq n_{i}-1$ for each $i$, $1 \leqq i \leqq k$, which produces the desired result.

Vertex-arboricity Ramsey numbers. The vertex-arboricity $a(G)$ of a graph $G$ is the minimum number of subsets into which the vertex set of $G$ may be partitioned so that each subset induces an acyclic subgraph. As with the chromatic number, the vertex-arboricity may be considered a coloring number since $a(G)$ is the least number of colors which may be assigned to the vertices of $G$ so that no cycle of $G$ has all of its vertices assigned the same color.

Our next result will establish a formula for the vertex-arboricity Ramsey number $a\left(n_{1}, n_{2}, \cdots, n_{k}\right)$, defined as the least positive integer $p$ such that for every factorization $K_{p}=G_{1} \cup G_{2} \cup \cdots \cup G_{k}, a\left(G_{i}\right) \geqq n_{i}$ for some $i, 1 \leqq i \leqq k$. Since $a\left(K_{n}\right)=\{n / 2\}$, it follows that $a\left(n_{1}, n_{2}, \cdots\right.$, $\left.n_{k}\right) \leqq r\left(2 n_{1}-1,2 n_{2}-1, \cdots, 2 n_{k}-1\right)$. In the proof of the following result, we shall make use of the (edge) arboricity $a_{1}(G)$ of a graph, which is the minimum number of subsets into which the edge set of $G$ may be partitioned so that the subgraph induced by each subset is acyclic. It is known (see $[1,4]$ ) that $a_{1}\left(K_{n}\right)=\{n / 2\}$.

THeOREM 2. For positive integers $n_{1}, n_{2}, \cdots, n_{k}$,

$$
a\left(n_{1}, n_{2}, \cdots, n_{k}\right)=1+2 k \prod_{i=1}^{k}\left(n_{i}-1\right) .
$$

Proof. In order to show that

$$
a\left(n_{1}, n_{2}, \cdots, n_{k}\right) \leqq 1+2 k \prod_{i=1}^{k}\left(n_{i}-1\right),
$$

we let $p=1+2 k \prod_{i=1}^{k}\left(n_{i}-1\right)$ and assume there exists a factorization $K_{p}=G_{1} \cup G_{2} \cup \cdots \cup G_{k}$ such that $a\left(G_{i}\right) \leqq n_{i}-1$ for each $i=$ $1,2, \cdots, k$. For each $i=1,2, \cdots, k$, there is a partition $\left\{U_{i, 1}, U_{i, 2}, \cdots\right.$, $\left.U_{i, n_{i}-1}\right\}$ of the vertex set $V\left(G_{i}\right)$ of $G_{i}$ such that the subgraph $\left\langle U_{i, j}\right\rangle$ of $G_{i}$ induced by $U_{i, j}$ is acyclic, $j=1,2, \cdots, n_{i}-1$. At least one of the sets $U_{1,1}, U_{1,2}, \cdots, U_{1, n_{1}-1}$, say $U_{1, m_{1}}$, contains at least $1+$ $2 k \prod_{i=2}^{k}\left(n_{i}-1\right)$ vertices. Thus, at least one of the sets $U_{2,1}, U_{2,2}, \cdots$, 
$U_{2, n_{2}-1}$, say $U_{2, m_{2}}$, contains at least $1+2 k \prod_{i=3}^{k}\left(n_{i}-1\right)$ vertices of $U_{1, m_{1}}$. Proceeding inductively, we arrive at subsets $U_{1, m_{1}}, U_{2, m_{2}}, \cdots$, $U_{k, m_{k}}$ such that $\bigcap_{i=1}^{t} U_{i, m_{i}}$ contains at least $1+2 k \prod_{i=t+1}^{k}\left(n_{i}-1\right)$ vertices, $1 \leqq t \leqq k-1$. In particular, $\bigcap_{i=1}^{k} U_{i, m_{i}}$, contains a set $U$ having $1+2 k$ vertices. For each $i=1,2, \cdots, k,\langle U\rangle$ is an acyclic subgraph of the graph $\left\langle U_{i, m_{i}}\right\rangle$. This implies that $a_{1}\left(K_{1+2 k}\right) \leqq k$, which is contradictory. Therefore, $a\left(G_{i}\right) \geqq n_{i}$ for at least one $i, 1 \leqq i \leqq k$.

The proof will be complete once we have verified that

$$
a\left(n_{1}, n_{2}, \cdots, n_{k}\right) \geqq 1+2 k \prod_{i=1}^{k}\left(n_{i}-1\right) .
$$

Let $r=\prod_{i=1}^{k}\left(n_{i}-1\right)$. We shall exhibit a factorization $K_{2 k r}=G_{1} \cup$ $G_{2} \cup \cdots \cup G_{k}$ such that $a\left(G_{i}\right) \leqq n_{i}-1$ for $i=1,2, \cdots, k$. We begin with $r$ pairwise disjoint copies of $K_{2 k}$, labeled $K_{2 k}^{1}, K_{2 k}^{2}, \cdots, K_{2 k}^{r}$. Since $a_{1}\left(K_{2 k}\right)=k$, it follows that $K_{2 k}=\bigcup_{i=1}^{k} F_{i}$, where each $F_{i}$ is an acyclic graph. We introduce the notation $F_{i l}$ to denote the $F_{i}$ contained in $K_{2 k}^{l}, l=1,2, \cdots, r$ and $i=1,2, \cdots, k$. With each of the $r k$-tuples $\left(c_{1}, c_{2}, \cdots, c_{k}\right), c_{j}=1,2, \cdots, n_{j}-1$ and $j=1,2, \cdots, k$, we identify a complete graph $K_{2 k}^{l}, l=1,2, \cdots, r$, in such a way that the identification is one-to-one. Then, for each $i=1,2, \cdots, k$ and $l=1,2, \cdots$, $r$, we associate with $F_{i l}$ the $k$-tuple identified with $K_{2 k}^{l}$. Define the graph $G_{i}, \quad i=1,2, \cdots, k$, to consist of the graphs $F_{i 1}, F_{i 2}, \cdots, F_{i r}$; in addition, each vertex of $F_{i s}$ is adjacent to each vertex of $F_{i t}$, $s, t=1,2, \cdots, r$, provided the $i$ th coordinate is the first coordinate in which their associated $k$-tuples differ (otherwise, there are no edges between $F_{i s}$ and $F_{i t}$ ). It is then seen that $K_{2 k r}=\bigcup_{i=1}^{k} G_{i}$. For each $i=1,2, \cdots, k$, define $V_{i, j}$ to be the set of all vertices $v$ such that $v$ is a vertex of an $F_{i l}$ whose associated $k$-tuple $\left(c_{1}, c_{2}, \cdots, c_{k}\right)$ has $c_{i}=j ; j=1,2, \cdots, n_{i}-1$. Then $\left\{V_{i, 1}, V_{i, 2}, \cdots, V_{i, n_{i^{-1}}}\right\}$ is a partition of $V\left(G_{i}\right)$ for which the subgraph $\left\langle V_{i, j}\right\rangle$ consists of $r /\left(n_{i}-1\right)$ pairwise disjoint copies of $F_{i}, j=1,2, \cdots, n_{i}-1$. Thus, $\left\langle V_{i, j}\right\rangle$ is an acyclic graph for each such $j$. Hence, $a\left(G_{i}\right) \leqq n_{i}-1$, $i=1,2, \cdots, k$.

\section{REFERENCES}

1. L. W. Beineke, Decompositions of complete graphs into forests, Magyar Tud. Akad. Mat. Kutato Int. Kozl., 9 (1964), 589-594.

2. J. E. Graver and J. Yackel, Some graph theoretic results associated with Ramsey's theorem, J. Combinatorial Theory, 4 (1968), 125-175.

3. R. E. Greenwood and A. M. Gleason, Combinatorial relations and chromatic graphs, Canad. J. Math., 7 (1955), 1-7.

4. C. St. J. A. Nash-Williams, Edge-disjoint spanning trees of finite graphs, J. London Math. Soc., 36 (1961), 445-450.

5. F. P. Ramsey, On a problem of formal logic, Proc. London Math. Soc., 30 (1930), 264-286. 
Received October 4, 1974. The second author's research was supported by the Research Foundation of the State of New York.

Western Michigan University

AND

Suny, College at Fredonia 



\section{PACIFIC JOURNAL OF MATHEMATICS}

EDITORS

RICHARD ARENS (Managing Editor)

University of California

Los Angeles, Calıfornia 90024

R. A. Beaumont

University of Washington

Seattle, Washington 98105
J. DugundJI

Department of Mathematics

University of Southern California

Los Angeles, California 90007

D. Gilbarg and J. Milgram

Stanford University

Stanford, California 94305

\section{ASSOCIATE EDITORS}
E. F. BECKENBACH
B. H. NeumanN
F. WOLF
K. YOSHIDA

\section{SUPPORTING INSTITUTIONS}

UNIVERSITY OF BRITISH COLUMBIA

CALIFORNIA INSTITUTE OF TECHNOLOGY

UNIVERSITY OF CALIFORNIA

MONTANA STATE UNIVERSITY

UNIVERSITY OF NEVADA

NEW MEXICO STATE UNIVERSITY

OREGON STATE UNIVERSITY

UNIVERSITY OF OREGON

OSAKA UNIVERSITY
UNIVERSITY OF SOUTHERN CALIFORNIA

STANFORD UNIVERSITY

UNIVERSITY OF TOKYO

UNIVERSITY OF UTAH

WASHINGTON STATE UNIVERSITY

UNIVERSITY OF WASHINGTON

AMERICAN MATHEMATICAL SOCIETY NAVAL WEAPONS CENTER 


\section{Pacific Journal of Mathematics}

\section{Vol. 55, No. $1 \quad$ September, 1974}

Robert Lee Anderson, Continuous spectra of a singular symmetric

differential operator on a Hilbert space of vector-valued functions . . . $\quad 1$

Michael James Cambern, The isometries of $L^{p}(X, K) \ldots \ldots \ldots \ldots \ldots . . \ldots$

R. H. Cameron and David Arne Storvick, Two related integrals over spaces of continuous functions ................................

Gary Theodore Chartrand and Albert David Polimeni, Ramsey theory and

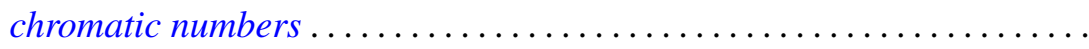

John Deryck De Pree and Harry Scott Klein, Characterization of collectively compact sets of linear operators ...................

John Deryck De Pree and Harry Scott Klein, Semi-groups and collectively compact sets of linear operators ....................... 55

George Epstein and Alfred Horn, Chain based lattices.............. 65

Paul Erdős and Ernst Gabor Straus, On the irrationality of certain series . . 85

Zdeněk Frolík, Measurable uniform spaces................... 93

Stephen Michael Gagola, Jr., Characters fully ramified over a normal

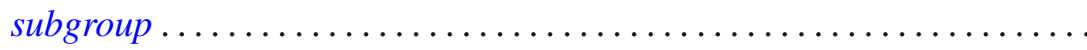

Frank Larkin Gilfeather, Operator valued roots of abelian analytic

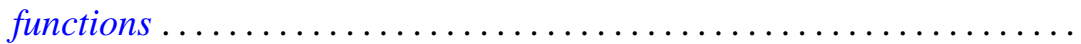

D. S. Goel, A. S. B. Holland, Cyril Nasim and B. N. Sahney, Best approximation by a saturation class of polynomial operators

James Secord Howland, Puiseux series for resonances at an embedded

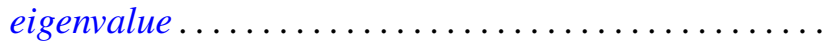

David Jacobson, Linear GCD equations .................

P. H. Karvellas, A note on compact semirings which are multiplicative

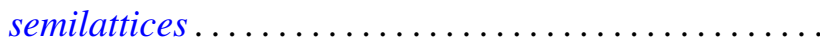

Allan Morton Krall, Stieltjes differential-boundary operators. II . .

D. G. Larman, On the inner aperture and intersections of convex sets

S. N. Mukhopadhyay, On the regularity of the $P^{n}$-integral and its application to summable trigonometric series ....... .

Dwight Webster Read, On $(J, M, m)$-extensions of Boolean algebras ....

David Francis Rearick, Multiplicativity-preserving arithmetic power series.

Indranand Sinha, Characteristic ideals in group algebras

Charles Thomas Tucker, II, Homomorphisms of Riesz spaces . . .

Kunio Yamagata, The exchange property and direct sums of indecomposable injective modules. 